\title{
Poscosecha de flores de corte y medio ambiente
}

\author{
Cut flowers postharvest and environment
}

Fernando de La Riva Morales ${ }^{1}$

\begin{abstract}
RESUMEN
Se ha determinado que un tercio de la vida de la flor cortada está influenciada por el ambiente de precosecha, mientras que los dos tercios restantes por el manejo y las condiciones reinantes después del corte. El transporte de flores a largas distancias se ha ido incrementando debido a que países como Kenia, Zimbabwe, Australia, Chile, Tailandia, Sudáfrica, Polonia, India y China se han ido incorporando a la producción florícola y cuentan con extensos programas para la exportación, sin embargo se encuentran distantes de los principales centros de comercialización y consumo como son Estados Unidos de Norteamérica, Europa y Japón. Lo anterior conlleva la necesidad de desarrollar tecnologías que permitan mantener la calidad de las flores cortadas, tales como conservación a bajas temperaturas, el uso de biocidas como el cloro o de preservantes químicos inhibidores del etileno (TSP), principal elemento causante de la maduración y senescencia de flores y frutos, o el empleo de técnicas mecánicas como el corte de los tallos bajo el agua, o la inmersión en ácido cítrico o aguas que contengan productos comerciales como Florissima o Pokon, entre otros. La calidad de los productos florales demandados por los consumidores europeos es extremadamente alta, los estándares de calidad de la Unión Europea están definidos en la norma 316/68 en la que se fijan los requerimientos mínimos para las flores cortadas y su tratamiento en postcosecha. En el presente trabajo se analizan las causas de la senescencia de las flores de corte y los preservantes no contaminantes para prolongar su vida útil en jarrón en forma respetuosa con el medio ambiente.
\end{abstract}

Palabras clave: senescencia, preservantes, contaminación, salud.

\begin{abstract}
It has been determined that a third of the life of cut flowers is influenced by pre-harvest environment, while the remaining two thirds for the management and the conditions after cutting. The transport of flowers over long distances has increased because countries like Kenya, Zimbabwe, Australia, Chile, Thailand, South Africa, Poland, India and China, have gone into production and have extensive flower for export programs, however distant from the main centers of trade and consumption as the United States of America, Europe and Japan. This implies the need to develop technologies to maintain the quality of cut flowers, such as low temperature storage, the use of chemical preservatives and biocides as chlorine, ethylene inhibitors (TSA), the main element that causes ripening and senescence of flowers and fruit, or the use of mechanical techniques such as cutting the stems under water, immersion in citric acid or water containing commercial products or Pokon, Florissima, among others. The quality of floral products demanded by European consumers is extremely high quality standards of the European Union are defined in the standard 316/68 laying down minimum requirements for cut flowers. This paper analyzes the causes of the senescence of cut flowers and clean preservatives to prolong their vase life as environmentally.
\end{abstract}

Key words: senescence, preservatives, contamination, health.

\section{Senescencia de las flores}

La senescencia de la flor se acelera cuando se separa de la planta, lo que determina que en pocos días la flor pierda su valor comercial. Se considera como longevidad de la flor el tiempo que ésta conserva sus cualidades decorativas, es decir, el tiempo que tardan en aparecer claros síntomas de marchitez. Este proceso de senescencia está programado genéticamente y controlado por la hormona etileno, produciéndose los cambios relacionados con él por la expresión de genes específicos (Van Alvorst y Bovy, 1995). Además, al inicio de la senescencia de determinadas especies de flor cortada se produce un ligero aumento de peso durante los primeros días desde la recolección y posteriormente éste desciende de forma muy acusada, coincidiendo con el inicio de la pérdida de peso fresco, también comienza a aumentar la tasa de producción de etileno. Por ello, los primeros síntomas de envejecimiento se

1 Dr. Ingeniero Agrónomo. Universidad de Tarapacá, Facultad de Ciencias Agronómicas. Departamento de Producción Agrícola. Casilla 6D, Arica Chile.E-mail: fdelariv@uta.cl

Fecha de Recepción: 12 Mayo, 2011.

Fecha de Aceptación: 03 Octubre, 2011. 
detectan cuando se inicia la producción de etileno, lo que coincide también con el descenso del peso fresco (Woodson y Lawton, 1988).

Esta producción de etileno es autocatalítica, lo que significa que el etileno producido estimula su propia síntesis, lo cual hace aumentar bruscamente su tasa de producción, hasta alcanzar un máximo, que seguidamente desciende en los dos últimos días de la senescencia. En las flores climatéricas es esta hormona la que inicia y regula los procesos que finalmente conducen a su muerte programada. Debido a la importancia del etileno en la senescencia de las flores climatéricas, se han realizado numerosos estudios utilizando inhibidores de las dos enzimas clave implicados en la biosíntesis de la hormona (ACC sintetasa y ACC oxidasa) o de su acción hormonal, compuestos que consiguen inhibir la producción de etileno y retrasar la senescencia (Wang y Woodson, 1989; Serrano et al., 1990; Van Alvorst y Bovy, 1995). Por otra parte, Li-Jen Liao (2001) indica que es bien conocido que la proliferación de bacterias en el agua del florero acorta la vida de las flores cortadas, por lo que la aplicación de productos biocidas es recomendada.

\section{Conservación de la flor cortada}

La vida de poscosecha de las flores de corte es limitada a menudo por una acumulación de bacterias en las soluciones para la hidratación de las varas florales (Havely y Mayak, 1981). Para aumentar la longevidad de la flor climatérica cortada, además de inhibir la biosíntesis de etileno, también es necesario mantener un aporte de agua adecuado a la flor, por lo que la disolución conservante debe tener compuestos que impidan la proliferación de microorganismos, que taponarían los vasos conductores, así como aportar a la flor una fuente nutritiva que satisfaga sus necesidades metabólicas. Algunas disoluciones diseñadas tienen una base común y que consiste en tampón cítrico-citrato, $\mathrm{pH} 4$, que contiene además sacarosa como fuente carbonada, para mantener un aporte energético a la flor, y Tritón X-100, como agente tensoactivo y humectante (Serrano et al., 1987).

La sacarosa contribuye a mantener el balance hídrico en la flor cortada, al provocar el cierre de los estomas (impidiendo así la pérdida inicial de agua), y además, favorece la retención de agua y solutos por las células, preservando la integridad de la membrana a través de procesos dependientes del metabolismo energético (De Stigter, 1981).

La adición de azúcar al agua del florero no sólo prolonga la vida de la flor sino que también promueve su apertura. Asimismo, la expresión del color de la flor es incrementada con el tratamiento de azúcar en algunas flores como claveles, rosas y Lisianthus. Los pigmentos de estas flores son principalmente antocianinas (Gilman y Steponkus, 1972; Parups y Molnar, 1972; Ichimura y Korenaga, 1998, Hojjati et al., 2007). Uddin et al., (2004) agregan que el uso de azúcar para promover el desarrollo de las flores y su pigmentación es una práctica común en la poscosecha de las flores cortadas. Al respecto, Ichimura y Korenaga (1998), Mayak y Dilley, 1976, Macnish et al., 2008 indican que la adición de azúcares al agua del depósito no sólo prolonga la vida útil de las flores sino que también promueve su apertura, mejora el color de algunas flores como el clavel y prolonga su vida en el florero, lo que está asociado con el mejoramiento en la absorción de agua. El requerimiento de carbohidratos fue demostrado en flores de corte de petunia (Weiss y Haley, 1991), de spandragon (Cantó et al., 1991) y del Lisianthus (Jamal et al., 2001). El uso de sacarosa al $4 \%$ más $50 \mathrm{mg} \mathrm{L}^{-1}$ de BA prolongó la vida en el florero e inhibió la senescencia en Eustoma (Kuang-Liang y Wen-Shan, 2002).

Los efectos del azúcar en la extensión de la vida en florero de las flores cortadas se considera que están asociados a la mejora del equilibrio de agua (Ichimura, 2007, Shimizu et al., 2007). Se requiere una gran cantidad de carbohidratos solubles para que se abran los capullos de la flor, los que actúan como sustratos para las membranas celulares y para la respiración, así como para sus características osmóticas. Puesto que la fuente de carbón en las flores de corte es limitada, la adición de azúcares tales como sacarosa y glucosa al agua del florero es altamente eficaz en promover la apertura de la flor (Downs et al., 1988; Paulin y Jamal, 1982).

Sin embargo, la solución nutritiva no consigue eliminar totalmente la contaminación microbiana, lo que constituye un problema durante la manipulación, comercialización y mantenimiento posterior de las flores en casa del consumidor. Para intentar eliminar este problema se han ensayado diferentes compuestos germicidas, encontrando que los amonios cuaternarios eran los que presentaban las mejores prestaciones. Para bloquear la síntesis de etileno, responsable de la senescencia de las flores 
climatéricas, se han utilizado diversos compuestos que actúan sobre las enzimas implicadas en la biosíntesis de esta hormona vegetal o sobre el receptor hormonal responsable, tras la unión con el etileno, de su acción fisiológica. Aunque todos estos compuestos presentan una alta efectividad, sus aplicaciones prácticas son muy limitadas debido esencialmente a su toxicidad para el consumidor y también por su elevado costo, por lo que su utilización ha quedado restringida a la investigación.

Otra posibilidad de inhibir la síntesis de etileno es bloquear el receptor hormonal, de forma que al impedir la unión de ambos compuestos se evita la acción fisiológica del etileno y se retrasa sensiblemente el inicio de los procesos de senescencia de la flor cortada. Algunos compuestos como el 2,5 norbonadieno y el diazociclopentadieno que bloquean al receptor hormonal no pueden utilizarse en la práctica por ser altamente cancerígenos.

En la actualidad para logar esta acción y prolongar la vida comercial útil de la flor ornamental cortada se está utilizando ión $\mathrm{Ag}^{+}$, aplicado bajo la forma de complejo con el ión tiosulfato (TSP). Las soluciones conservantes presentes en el mercado para los tratamientos en continuo o en pulsaciones contienen este complejo químico. Sin embargo, también se está cuestionando el empleo del ión $\mathrm{Ag}^{+}$, por su toxicidad para el consumidor y porque la eliminación de las soluciones utilizadas presenta un grave problema al ser altamente contaminante y muy agresivo con el medio ambiente debido a la permanencia del catión plata en el suelo y en las aguas subterráneas por períodos prolongados pudiendo pasar a los sistemas de agua potable llegando finalmente a ser absorbidos por los seres humanos (Nell, 1992). Por ello su uso se ve limitado por las normas de la Unión Europea que lo restringe por razones de contaminación ambiental.

El tratamiento con sulfato de aluminio, $\mathrm{Al}_{2}\left(\mathrm{SO}_{4}\right)_{3}$ también ha sido recomendado para mantener la vida en el florero de varias flores de corte (De Stigter, 1981; Van Doorn, 1997; Ichimura y Ueyama, 1998). Esto se basa en parte en su acción como agente antimicrobiano en la solución del florero (Havely y Mayak, 1981). De Stigter (1981), por ejemplo, encontró que tratando rosas con $\mathrm{Al}^{+3}$ obtuvo una mejor calidad que en el control con agua sola. También Ichimura y Korenaga (1998) indican que la vida en florero de Lisianthus se prolonga notablemente con la adición de sulfato de 8-hydroxyquinolina. Su vez, Li-Jen Liao et al., (2001) encontraron que la vida en jarrón de las flores de Lisianthus se prolongó por 15 días usando $150 \mathrm{mg} \mathrm{L}^{-1}$ de sulfato de aluminio, a diferencia del control sólo con agua, cuya duración fue de 8 días. Havely y Kofranek (1984) señalan que otras opciones son soluciones con agua desionizada con $10 \%$ de azúcar, ácido cítrico y agentes antimicrobianos, los que aplicados por 24 horas han permitido prolongar en más de13 días la vida de poscosecha del Lisianthus, pero con el resultado de que todas las flores abran al mismo tiempo.

Otros compuestos antimicrobianos se han descrito para ampliar la vida en jarrón de las flores de corte, tales como clorina (ej. hipoclorito de sodio $[\mathrm{NaOCl}]$ ), que cuando es utilizada con el agua del florero puede reducir el número de bacterias y aumentar longevidad de la flor (Havely y Mayak, 1981). La eficacia del hipoclorito de sodio es relativamente pobre entre las soluciones acidificantes (ej. pH 3-4) que se recomiendan generalmente para la hidratación de las flores de corte (Nowak y Rudnicki, 1990; White, 1999), y tiene un bajo rendimiento como agente desinfectante con la materia orgánica. Además, puede ser fitotóxico para las flores (van Doorn y otros, 1990; Knee, 2000).

El hipoclorito de sodio se obtuvo por primera vez en Javel, barrio periférico de París, por el químico francés Bertholet, que en 1785 experimentó la fórmula descubriendo su utilización y las posibles aplicaciones desinfectantes. A finales del siglo XIX, momento en que Louis Pasteur descubre que los microorganismos son los causantes de las enfermedades, la clorina tuvo el momento de máximo reconocimiento, gracias a sus propiedades como activo agente antiséptico. Se entiende por clorina la solución de hipoclorito con un contenido de cloro activo no inferior a $35 \mathrm{~g} / \mathrm{l}$ ni superior a 100 g/l. (Amiclor, 2005).

Los vapores de la clorina producen la irritación a la piel y a las vías respiratorias. Algunos trihalometanos, tales como cloroformo $\left(\mathrm{CHCl}_{3}\right)$ y bromodi-clorometano $\left(\mathrm{CHBrCl}_{2}\right)$, pueden ser carcinógenos, mutágenos, teratogénicos o tóxicos según numerosos estudios. Además, se ha encontrado una relación directa entre estas sustancias y las incidencias del cáncer de vejiga y las anomalías congénitas (Chu et al., 1982; Carpenter y Beresford, 1986; Maxwell et al., 1991; Dunnick y Melnick, 1993; Pilotto, 1995; Villanueva et al., 2000; Ritter et al., 2002). La Agencia de Protección Ambiental (EPA, 1998) ha establecido un límite $60 \mu \mathrm{g} \mathrm{L} \mathrm{L}^{-1}$ para 
el cloroformo y $40 \mu \mathrm{g} \mathrm{L} \mathrm{L}^{-1}$ para bromoformo como riesgo medio de contraer el cáncer. Los valores aceptables máximos aconsejados por la Unión Europea para los trihalometanos totales establece el límite en $100 \mu \mathrm{gL}^{-1}$ (EECD, 1997). Aparte del problema de la higiene ambiental, la desinfección con cloro también tiene un efecto limitado sobre la reducción de microorganismos en la superficie de las frutas y verduras (Sapers, 2001).

\section{Productos respetuosos con el medio ambiente}

Existe la necesidad de utilizar productos biodegradables que sean respetuosos con el medio ambiente, como productos orgánicos: sacarosa, ácido peroxiacético o agua oxigenada, entre otros. Geenspan y Margulies (1950) patentaron el ácido peracético en 1950 para tratar las frutas y verduras para reducir la proliferación de bacterias y de hongos. También había sido utilizado para otros propósitos tales como desinfestaciones de bulbos y contra nematodos (Hanks y Linfield, 1999) y para la prevención de otras enfermedades hortícolas mediante la desinfección del suelo y la limpieza de los equipos de riego (Larose y Abbot, 1998).

Hay una larga historia del uso en el campo experimental del ácido peracético como fungicida/ bactericida, no obstante su eficacia se ha determinado sólo recientemente. Hei (2000) divulgó su utilidad al control de enfermedades hortícolas en campo del tejido vegetal, las semillas, las frutas, los medios y los envases. El perácido mezclado o los sistemas peracéticos se hacen con el ácido peracético (PAA), el peróxido de hidrógeno y el ácido acético. Esta mezcla no es virtualmente afectada por los cambios de temperatura, incluso en presencia de materia orgánica, cuando oxida el material orgánico, finalmente se descompone en anhídrido carbónico y agua. (Rodgers et al., 2004), constituyéndose en productos altamente amigables con el medio ambiente.

En un estudio realizado por de La Riva, et al. (2009), en postcosecha de flores de lisianthus tratadas con agua con azúcar al $3 \%$ sola, y con hipoclorito de sodio $\left(1 \mathrm{ml} \mathrm{L}^{-1}\right)$ y con peróxido de hidrógeno $\left(0,1 \mathrm{ml} \mathrm{L}^{-1}\right)$, observaron que el efecto del azúcar en la solución preservante se vio potenciado con los biocidas debido a que las flores se apreciaron con mejor color y hojas más turgentes hasta el final del ensayo, así como también presentaron una mayor duración en florero, con medias de 26 días en ambos tratamientos, pero presentando los dos una alta diferencia con el tratamiento sólo con azúcar, en que se obtuvo una duración media de 12 días. Sin embargo, concluyeron que el uso del peróxido de hidrógeno por ser más amigable con el medio ambiente, al descomponerse en agua y oxígeno libre sería más recomendable, con resultados similares a los del hipoclorito de sodio.

Las regulaciones españolas permiten el uso del peróxido de hidrógeno y del ácido peracético en el agua de consumo y de lavado de las frutas y verduras (AENOR, 2005; BOE, 2003). La Agencia de Protección Ambiental (EPA, 1998) autoriza el uso de los añadidos al agua de lavado a base de ácido peroxiacético (PAA) para las frutas y verduras (ej. Tsunami ${ }^{\circledR} 100$, Ecolab). Además, es bien sabido que PAA es un oxidante y un desinfectante que es más eficiente que el dióxido de la clorina o del chorine (Kitis, 2004).

\section{Referencias Citadas}

\footnotetext{
AENOR

2005 Asociación Española de Normalización y Certificación. UNE155000. Frutas y hortalizas frescas. ENOR, 2005.

Amiclor

2006 Información sobre el cloro. Organización de usuarios y trabajadores de la química del cloro. Consulta electrónica 31/01/2009, disponible en:

http://www.amiclor.org/opciones/info_clor.shtml BOE

2003 RD/140/2003. No 45. Boletín Oficial del Estado. Spain. pp. $7228-7245$.

Carpenter L.M.; Beresford, S.A.

1986 Cancer mortality and type of water source: findings from a study in the UK. International Journal of Epidemiology 5:312-320 .
}

Chu, I.; Villeneuve, D.C.; Secours, V.E.; Becking, G.C. and Valli,V.E. 1982 Toxicity of trihalomethanes: the acute and subacute toxicity of chloroform, bromodichloromethene, chlorodibromomethene and bromoform in rats. Journal of Environmental Science and Health 17: 205-224.

De La Riva, M.; Mazuela, P.; Álvaro, J. y Urrestarazu, M.

2009 Treatment with Peracetic Acid Extends the Vase Life of Lisianthus (Eustoma grandiflorum) Flowers. HortScience 44 (2):418-420. 2009.

De Stigter, H.C.M.

1981 Effects of glucose with 8-hydroxyquinoline sulfate or aluminum sulfate on the water balance of cut 'Sonia' roses. Z. Pflanzenphysiol. 101: 95-105.

Downs, C.; Reihana, M.; Dick, H.

1988 Bud-opening treatments to improve Gypsophila quality after transport. Sci. Hort. 34: 301-310. 
Dunnick, J.K. and Melnick, R.L.

1993 Assessment of the carcinogenic potential of chlorinated water: Experimental studies of chlorine, chloramines and Trihalomethanes, J. Natl. Cancer Inst. 85:817-822.

EECD

1997 European Economic Community Directive. Ammended proposal for a Council Directive concerning the quality of water intended for human consumption-common position. In: Proceedings of the Council of the European Union, Directive 80/778/EEC, Com (97) 228 Final 95/0010 SYN, Brussels.

EPA

1998 Food and Drugs Administration. Pub. N 98-18287 21 CFR Part 101. U.S. Health and Human Services. Springfield, VA.

Geenspan, F. P. and Margulies, P.H.

1950 Treatment of row plant tissue. US. Patent 2512640. Buffalo Electro-Chemical Co.

Gilman, K. F.; Steponkus, P.L.

1972 Vascular blokage in cut roses. J. Am. Soc. Hort. Sci.; 97: 662-667.

Hanks, G.R.; Linfield, C.A.

1999 Evaluation of a peroxyacetic acid disinfectant in hot-water treatment for the control of basal rot (Fusarium oxysporum f. sp. narcissi) and stem nematode (Ditylenchus dipsaci) in narcissus. J. Phytopathol. 147(5): 271-279.

Havely A.H., and Mayak S.

1981 Senescence and postharvest physiology of cut flowers. Part 2. Hort. Rev. 3:59-153.

Havely A.H, and Kofranek, A.M.

1984 Evaluation of lisianthus as a new flower crop. HortScience 19 (6):845-847.

Hei, R.D.P.

2007 Peroxy acid treatment to control pathogenic organisms on gowing plants. US Patent 6, 024, 986.

Hojjati,Y.; Khalighi, A.; Farokhzad, A. R.

2007 Chemical Treatments of Eustoma Cut Flower Cultivars fo Enhaced Vase Life. Journal Agriculture of Social Science. 1813-2235 / 2007 / 03. 75-78.

Ichimura, K.; Korenaga, M.

1998 Improvement of vase life and petal color expression in several cultivars of cut eustoma flowers using sucrose with 8-hydroxyquinoline sulfate. Bull. Nat. Res. Veg Ornam. Plants \& Tea. Japan. 13: 31-39.

Ichimura, K.; Ueyama, S.

1998 Effects of temperature and application of aluminum sulfate on the postharvest life of cut rose flower. Bull. Natl. Res. Inst. Veg.; Ornam. Plants.

Ichimura, K.

2007 Effects of sucrose treatment on the vase life of various cut flowers. Bull. Natl. Res. Inst. Veg.; Ornam. Plants.

Jamal Uddin, A.F.M.; F. Hashimoto, M. Kaketani, K. Shimizu, K.; Sakata, Y.

2001 Analysis of light and sucrose potencies on petal coloration and pigmentation of lisianthus cultivars (in vitro). Scientia Horticulturae 89: 75-84.

Kitis, M.

2004 Disinfection of wastewater with peracetic acid: a review. Environment International 30: 47-55.

Knee, M.

2000 Selection of biocides for use in floral preservatives. Postharvest Biol. Technol. 18: 227-234.
Kuang-Liang, H.; Wen-Shaw, Ch.

2006 BA and sucrose increase vase life of cut Eustoma flowers. HortScience 37 (3): 547-549.

Larose, R.N.; Abbot, M.N.

1998. Method for control of horticulture diseases, and decontamination of plant tissue. US Patent 5, 723, 406.

Li-Jen Liao, Yu-Han Lin, Kuang-Liang Huang, Wen-Shaw Chen 2001 Vase life of Eustoma grandiflorum as affected by aluminium sulfate. Bot. Bull. Acad. Sin. 42: 35-38.

Macnish, A.J.; Leonard, R.T.; Nell, T. A.

2008 Treatment with chlorine dioxide extends the vase life of selected cut flowers. Postharvest Biology and Technology (In press).

Maxwell, N.I.; Burmaster, D.E.; Ozonoff, D.

1991 Trihalomethanes and maximum contaminant levels: the significance of inhalation and dermal exposures to chloroform in household water. Regulatory Toxicology and Pharmacology 14: 297-312.

Mayak, S.; Dilley, D.

1976 Effect of sucrose on response of cut carnation flowers to kinetin, ethylene and abscisic acid. J. Am. Soc. Hort. Sci. 101: 583-585.

Nell, T.

1992 Taking silver safely out of the longevity picture. Groven Talks Magazine. June 92. p.23-26.

Nowak, J.; Rudnicki, R.M.

1990 Postharvest Handling and Storage of Cut Flowers, Florist Greens, and Potted Plants. Chapman and Hall, London. 187 p.

Parups, E.; Molnar, J.

1972 Histochemical study of xilem blockage in cut roses. J. Am. Soc. Hort. Sci.; 97, 532-534.

Paulin, A.; Jamal, C.

1982 Development of flowers and changes in various sugar during opening of cut carnations. J. Am. Soc. Hort. Sci., 107, 258-261.

Pilotto, L. S.

1995 Disinfection of drinking water, disinfection by products and cancer. What about Australia, Aust. J. Public Health 19: 89-93.

Ritter, L.; Solomon, K.; Sibley, P.; Hall, K.; Keen, P.; Mattu, G. and Linton B.

2002 Sources, path-ways, and relative risks of contaminant in surfacewater and groundwater. A perspective prepared for the Walkerton inquiry. J. Toxicol. Environ. Health 65: $1-142$.

Rodgers, S.T.; Cash, J.N.; Siddiq M.; Ryser, E.T.

2004 A comparison of different chemical sanitizers for inactivating Escherichia coli O157:H7 and Listeria monocytogenes in solution and on apples, lettuce, strawberries and cantaloupe. Journal of Food Protection 67: 721-731.

Sapers, G.M.

2001 Efficacy of washing and sanitizing methods for disinfection of fresh fruits and vegetable products. Food Technology and Biotechnology 39: 305-311.

Serrano, M.; Rosauro, J.; Del Rio, J. A.; Acosta, M.

1987 Conservación de la flor cortada de clavel (Dianthus caryophyllus, L. Cv. Arthur). 1. Uso de disoluciones conservadoras. Anales de Biología 14 (Biología General, 3): 39-44.

Serrano, M.; Romojaro, F.; Casas, J. L.; Del Río, J.; Acosta, M. 1990 Action and mechanisme of-aminoisobutyric acid as a retardant of cut carnation senescence. Scientia Horticulturae. 4: 127-134. 
Shimizu, H.; Ichimura, K.

2007 Effect of Relative Humidity and Sucrose Concentration of Leaf Injury and Vase Life During Sucrose Pulse Treatment in Cut Eustoma Flowers. Hort. Res. (Japan) 6: 301-305.

Uddin, J.; Hashimoto, F.; Shimizu, K.; Sakata, Y.

2004 Monosaccharides and chitosan sensing in bud growth and petal pigmentation in Eustoma grandiflorum (Raf.) Shinn. Scientia Horticulturae 100 (2004) 127-138.

Van Alvorst, A. C.; Bovy, A. G.

1995 The role of ethylene in the senescence of carnation flowers, a review. Plant Growth Regulation 16: 43-53.

Van Doorn, W.G.; de Witte Y.; Perik, R.R.J.

1990 Effect of antimicrobial compounds on the number of bacteria in stems of cut rose flowers. J. Appl. Bacteriol. 68: 117-122.

Villanueva, C. M.; Kogevinas, M. and Grimalt J.O.

2000 Cloración del agua potable en España y cáncer a la vejiga. Gac. Sanit. 15: 48-53.
Wang $\mathrm{H}$ y Woodson, W. R.

1989 Reversible inhibition of ethylene action and interruption of petal senescence in carnation flowers by norbornadiene. Plant Physiology 1989; 89: 434-438.

Weiss, D.; Haley, A. A.

1991 The role of light reactions in the regulation of anthocyanin synthesis in petunia corollas. Physiol. Plant 81:127-133.

White, G.C.

1999 Handbook of Chlorination and Alternative Disinfectants, 4th edition. John Wiley \& Sons, New York.

Woodson, W. R.; Lawton, K. A.

1988 Ethylene-induced gene expression in carnation petals. Relationship to autocatalytic ethylene production and senescence. Plant Physiol. 87: 498-503. 